\title{
ANÁLISE BIBLIOMÉTRICA SOBRE MODELOS DE LOCALIZAÇÃO DE FACILIDADE COM APLICAÇÃO NA LOGÍSTICA REVERSA ${ }^{1}$
}

\author{
Isabela Nunes Friginia ${ }^{*}$, Gisele de Lorena Diniz Chaves ${ }^{a}$, Glaydston Mattos Ribeiro ${ }^{b}$ \\ aDepartamento de Engenharias e Tecnologias, Centro Universitário Norte do Espírito Santo \\ Universidade Federal do Espírito Santo - UFES, Vitória-ES, Brasil

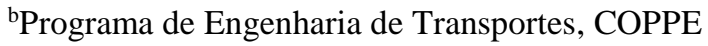 \\ Universidade Federal do Rio de Janeiro - UFRJ, Rio de Janeiro-RJ, Brasil
}

Recebido 02/02/2017, aceito 19/07/2017

\begin{abstract}
RESUMO
Este artigo tem como objetivo realizar um levantamento das publicações que fazem referência ao estudo do modelo de localização de facilidade com aplicação na logística reversa, no sentido de avaliar as publicações existentes e identificar orientações para pesquisas futuras. Para a pesquisa, foi utilizado o Portal Capes A pesquisa identificou os autores, periódicos, pares de documentos e instituições de pesquisas mais importantes neste tema. Além da análise descritivas, foi realizada uma análise bibliométrica de citação e cocitação por meio do software CiteSpace II. Dentre os 30 periódicos envolvidos nesta pesquisa, a revista de maior destaque em relação à temática é a Resources, Conservation and Recycling. As parcerias de pesquisa que estão concentradas com maior destaque nos EUA e no Canadá, que são também os países que mais publicam sobre o tema. Os resultados mostram que apesar das primeiras publicações se iniciarem em 1996, este tema teve suas publicações aumentadas significativamente a partir de 2007, evidenciando que o tema estudado é um campo emergente.
\end{abstract}

Palavras-chave: Modelos de localização, Logística reversa, Análise bibliométrica.

\begin{abstract}
This article aims to survey publications that refer to the study of the facility location model applied to reverse logistics, to evaluate existing publications and identify guidelines for future research. The databases used were the Capes Portal and Web of Science. The research identified the most important authors, journals, co-citations and research institutes in this area. In addition to the descriptive analysis, a bibliometric analysis of citations and co-citations using CiteSpace II software was carried out. Among the 30 journals used in this research, the most prominent journal addressing the topic is Resources Conservation and Recycling. Research partnerships are concentrated most notably in the US and Canada, which are also the countries that publish most on the subject. The results show that although the first publications on this topic first appeared in 1996, they increased significantly from 2007 showing that the studied subject is an emerging field.
\end{abstract}

Keywords: Facility location model, Reverse logistics, Bibliometric analysis.

*Autor para correspondência. E-mail: isa.belafrigini@gmail.com.

DOI: $10.4322 /$ PODes.2017.010 


\section{Introdução}

O aumento da importância da logística reversa vem também da crescente preocupação com o meio ambiente, devido a fabricação de produtos com ciclos de vida reduzidos, exigindo que as empresas se responsabilizem por seus produtos após a venda. Motivados também pela legislação brasileira, faz-se necessário dar uma destinação adequada a produtos que foram descartados, tanto aqueles que foram consumidos até o final de sua vida útil quanto aqueles que foram descartados sem atingir o fim de sua vida útil, em outras palavras por não servirem mais (Ferri et al., 2015). Com isso, é necessário ter em vista um planejamento do que fazer com o descarte dos produtos para garantir que esses sejam adequadamente descartados, aproveitados ou reparados.

Este planejamento envolve os estudos de localização de facilidades que podem ser, por exemplo, hospitais, escolas, centros de coleta e locais para a recuperação ou remanufatura (Melo et al., 2009). As decisões de localização geram grande interesse por indicarem melhores locais para alocação desde indivíduos até empresas e envolvem o caráter estratégico destas facilidades e seus efeitos na economia. A decisão no local de implantação implica na habilidade da empresa de competir no mercado, mas envolve também tomar decisões que implicam em fatores econômicos como poluição, congestionamentos, desenvolvimento econômico, entre outros (Drezner e Hamacher, 2002).

Para Fleischmann et al. (1997) as decisões de localização de uma instalação significam selecionar o mais adequado entre os potenciais locais previamente identificados definindo o número ideal dessas instalações e suas reais capacidades, facilitando dessa forma o fluxo do produto entre as mesmas. Modelos de localização têm sido bastante utilizados para auxiliar em problemas e decisões relacionados a logística reversa (Ghiani et al., 2014; Ferri et al., 2015). As redes de logística reversa definem o número, local e faixas de capacidade de centros de coleta, reaproveitamento e descarte de produtos, bem como define o fluxo de produtos e materiais entre eles (Ramezani et al., 2013; Pishvaee et al., 2010).

Em função do crescimento de publicações sobre este tema, torna-se relevante fazer um levantamento da produção científica, de modo a verificar quais são as referências, autores e periódicos mais destacados nesse tema e qual a conexão existente entre essas referências. Este artigo tem como objetivo analisar a literatura sobre a modelos de localização de facilidades relacionados com a logística reversa.

No caso das publicações científicas, pode-se pretender conhecer todos os autores que trabalharam em determinado assunto; os periódicos que publicados, as referências utilizadas, dentre outras variáveis de interesse, além de cruzar essas propriedades A finalidade da análise bibliométrica é o estudo das referências bibliográficas e das publicações, sendo um dos instrumentos básicos no estudo dos fenômenos da comunicação científica (Cardoso et al., 2005; Kobashi e Santos, 2008). Sua importância é atribuída pela característica de um método útil para mensurar a repercussão e impacto de determinados autores ou periódicos, permitindo que se conheçam as ocorrências de variação e suas tendências.

O artigo está dividido da seguinte forma: na Seção 2, apresenta-se o referencial teórico, destacando as definições e as aplicações da logística reserva, bem como sua relação com localização de facilidades; na Seção 3, as etapas e métodos de pesquisa utilizados neste trabalho são apresentados com intuito de analisar e avaliar as produções científicas referentes ao modelo de localização de facilidade com aplicação na logística reversa; na Seção 4, os resultados obtidos nesta pesquisa são apresentados e discutidos em três vertentes: análise bibliométrica das publicações, análise sistêmica das publicações e análise das pesquisas futuras propostas nas publicações; na Seção 5 as considerações finais acerca deste trabalho foram apresentadas.

\section{Referencial Teórico}

A logística é vista como o gerenciamento do fluxo de matérias do seu ponto de aquisição até o seu consumo. No entanto, Lacerda (2003) destaca que existe um fluxo de material 
proveniente do ponto de consumo em direção ao ponto de origem. Tal fluxo é gerenciado por meio da logística reversa.

A logística reversa, dentre inúmeras definições, pode ser compreendida como uma atividade responsável pelo planejamento e gerenciamento do fluxo reverso de produtos na cadeia produtiva (Rogers e Tibben-Lembke, 1999). Leite (2009), de forma mais especifica, descreve que a logística reversa é a área da logística empresarial que planeja e controla o fluxo e as informações logísticas ligadas ao retorno dos produtos após serem utilizados ou descartados, por meio dos canais de distribuição reverso.

O processo de logística reversa está ligado a inúmeras razões, como questões ambientais, redução de custos, vantagem competitiva e o ciclo de vida dos produtos. Para Fuller e Allen (1995) existem seis fatores que levam a aplicação da logística reversa: econômicos, governamentais, responsabilidade corporativa, tecnológicos, logísticos e sociais. Muitas empresas passaram a adotar a logística reversa como estratégia, montando redes reversas para agregar valor em toda cadeia.

No problema de concepção da rede de logística reversa, três tipos de decisões estão envolvidos: a primeira refere-se à localização de facilidades locais, a segunda diz respeito ao fluxo de matéria e informações entre estas entidades e a terceira é o investimento em equipamentos em cada uma das instalações. Stadtler (2005) afirma que na literatura, estas três categorias das decisões referem-se aos problemas de localização de facilidades, alocação e capacidade.

De acordo com Melo et al. (2009), há dois conjuntos de instalações dentro logística reversa: centros de coleta, que são instalações que recebem produtos usados e os locais para a recuperação ou remanufatura, em que os produtos são recondicionados ou remanufaturados. Além disso, um terceiro conjunto de instalações envolvidas em redes de logística reversa inclui os locais para o descarte final dos produtos, isto é, locais onde os produtos ou materiais são incinerados ou descartados (De Brito, 2004; Ramezani et al., 2013; Pishvaee et al., 2010). Neste contexto, a estrutura de rede de distribuição pode ser expandida para incluir o fluxo de retorno a partir de clientes para os locais de reparação ou destino final adequado (Ferri et al., 2015).

\section{Etapas e Método de Pesquisa}

Com intuito de analisar e avaliar as produções científicas referentes ao modelo de localização de facilidade com aplicação na logística reversa, realizou-se a análise bibliométrica. Segundo Macias-Chapula (1998) esta pesquisa se preocupa em estudar a mensuração da produção, disseminação e uso da informação registrada. No caso das publicações científicas, pode-se pretender conhecer todos os autores que trabalharam em determinado assunto; os periódicos que publicaram sobre esse assunto; os autores considerados referência no assunto; os autores desse assunto que publicaram em determinado periódico, dentre outras variáveis de interesse, além de cruzar essas propriedades. Esta análise permite "computar dados para comparar e confrontar os elementos presentes em referências bibliográficas de documentos representativos das publicações" (Kobashi e Santos, 2008, p. 109).

Cardoso et al. (2005) dizem que uma das formas de avaliar a produção científica em determinada área do conhecimento é pela análise bibliométrica. Seu objeto é o estudo das referências bibliográficas e das publicações, sendo um dos instrumentos básicos no estudo dos fenômenos da comunicação científica. Sua importância é atribuída pela característica de um método útil para mensurar a repercussão e impacto de determinados autores ou periódicos, permitindo que se conheçam as ocorrências de variação e suas tendências.

A pesquisa pode ser compreendida como quantitativa, pelas características de enumerar e medir, e qualitativa, pelas características de compreender e explicar. Segundo Kobashi e Santos (2008), o conhecimento qualitativo pode ser objetivado por relações quantificadas, provindas da aplicação de técnicas bibliométricas. Um conhecimento qualitativo não elimina a quantidade, mas procura-se tomar a medida como meio para compreender e explicar, de modo a quebrar a clivagem entre o modo quantitativo e o modo qualitativo de analisar objetos (Kobashi e Santos, 2008). Ou seja, os indicadores bibliométricos são caracterizados como quantitativos quando 
representam o processo avaliativo e como qualitativos quando possuem, por exemplo, a avaliação por pares, sendo utilizada, em geral, para julgar propostas de pesquisa, avaliar grupos de pesquisa e artigos científicos, entre outros aspectos.

Para a escolha do método da tabulação dos dados, realizou-se uma pesquisa com base em diferentes autores que utilizaram distintos procedimentos para a pesquisa bibliométrica. Penteado Filho et al. (2002) acredita que a bibliometria deve ser utilizada como um processo de tratamento automatizado da informação, transformando grande quantidade de dados de difícil análise em indicadores de alto valor agregado. O mesmo autor ressalta ainda que apenas a busca pelos dados nas pesquisas eletrônicas não é suficiente para uma análise bibliográfica, até porque essas bases de dados foram desenvolvidas para identificação e localização de documentos e não para análise.

Leeds (2000) cita as principais etapas no tratamento dos dados, começando pela busca das informações necessárias para contemplar a pesquisa, identificando suas necessidades; preparação dos dados; tratamento bibliométrico e análise dos resultados. Na etapa de tratamento bibliométrico são utilizadas tecnologias de análise bibliométrica, ou seja, são utilizados softwares que têm como princípio básico a representação das relações entre os elementos constituintes de um grupo de dados por meio de uma rede. Penteado Filho et al. (2002) alegam que o processo contínuo de transformação de dados brutos em conhecimento estratégico, tem crescido exponencialmente. A própria necessidade das empresas em obter conhecimento da sua cadeia de produção tem levado a um uso intensivo de tecnologias da informação disponibilizadas pela indústria de software em todo o mundo.

Desta forma, é notável perceber que a metodologia que deve seguir para a realização de uma pesquisa é, como enfatiza Leite Filho e Siqueira (2007, p.107), escolher a base de dados que obtenha o máximo de exemplares eletrônicos disponibilizados para pesquisa; coletar e organizar os dados em um banco de dados; tratar, comparar e analisar os dados por meio de um software que seja apropriado para a pesquisa em questão e que tabule com eficiência os dados coletados. Sendo assim, as etapas utilizadas para a condução desta pesquisa por bibliometria são apresentadas na Figura 1.

Figura 1: Etapas da análise bibliométrica.

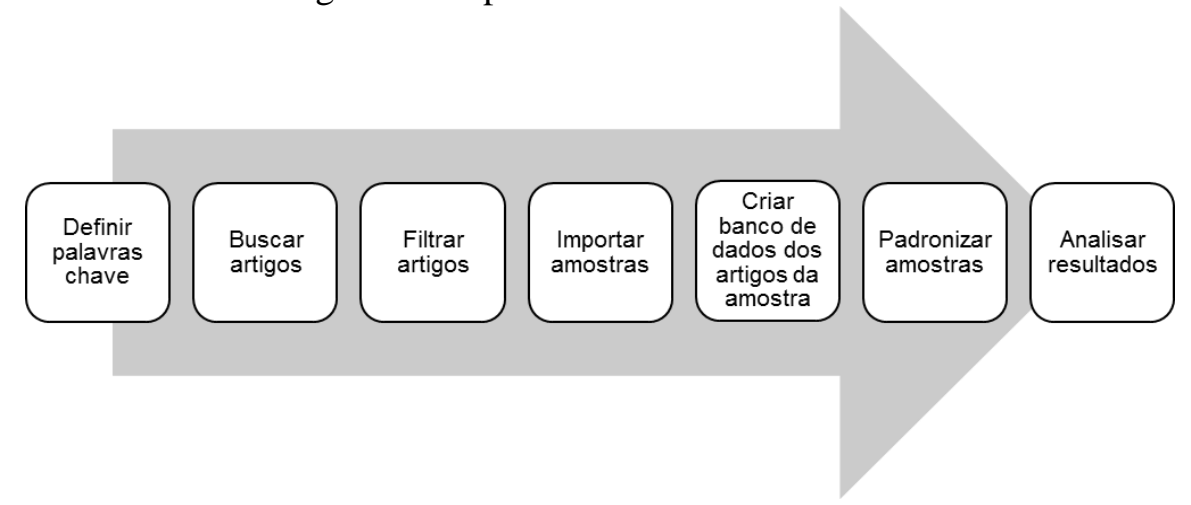

Fonte: Elaborada pelos autores.

Para iniciar a busca dos artigos desta pesquisa foi utilizado o Portal de Periódicos da Coordenação de Aperfeiçoamento de Pessoal de Nível Superior (Capes), que é uma biblioteca virtual que engloba muitas produções cientificas internacionais e as concede para as instituições de ensino e pesquisa no Brasil. Largamente utilizado pela comunidade cientifica para a divulgação de pesquisas, ele possui um acervo com mais de 37 mil títulos com texto completo, 126 bases referenciais, 11 bases dedicadas exclusivamente a patentes, além de livros, enciclopédias e obras de referência, normas técnicas, estatísticas e conteúdo audiovisual (Portal de Periódicos, 2015).

Para a seleção das referências, foram pesquisados em artigos apenas internacionais as palavras-chave que possuem grande relevância nos temas abordados, logística reversa e modelos de localização, sendo elas: reverse logistics, facility location, facility model, recycl, 
recover, refurbish e remanufactu. A procura utilizando esses termos foi realizada em 30 de abril de 2015, por meio da busca avançada do Portal Capes, onde foram selecionados os artigos publicados no maior espaço de tempo que o site oferece, de 1970 a 2015.

A busca avançada consiste de dois espaços localizadores para se fazer a pesquisa. No primeiro, foram colocadas as palavras-chave que seriam encontradas no campo "título" e, no segundo, as palavras-chave encontradas no campo "assunto" dos artigos. Para cada título abordado, mudou-se sete vezes a pesquisa no campo "assunto", para que a rede de ligação das palavras-chave fosse ampliada, garantindo que todos os artigos com estas palavras de interesse fossem obtidos.

Foram selecionados apenas os artigos que possuíam as palavras-chave tanto no título do artigo quanto no assunto do artigo, ou seja, no corpo do texto, resultando na amostra de 1676 artigos. Porém, foram contempladas apenas as publicações que passaram pela avaliação por pares, resultando em uma amostra final de 948 artigos. Após a definição dessa amostra procedeu-se com a importação dos artigos, para a criação de um banco de dados.

À medida em que os artigos foram sendo importados do Portal Capes, percebeu-se que haviam artigos que se repetiam, o que já era esperado devido à similaridade dos assuntos abordados. Portanto, a etapa seguinte consistiu na padronização dos dados, por meio da eliminação de artigos em duplicidade, o que resultou em 318 artigos. Destes, apenas 64 artigos foram selecionados como parte do escopo do trabalho, sendo todos referentes ao estudo do modelo de localização de facilidades com aplicação na logística reversa. Os demais artigos foram descartados em função de envolver somente os problemas de localização de facilidade ou somente a logística reversa.

$\mathrm{Na}$ realização da análise bibliométrica foi utilizado o software CiteSpace III, uma aplicação Java disponível gratuitamente para a realização de análises sobre as produções científicas Chen (2006). O software CiteSpace III foi escolhido pois, de acordo com Chen (2006), ele é capaz de gerar mapas a partir das produções cientificas, permitindo mapear e visualizar novas tendências na literatura científica, detectando pontos de viragem intelectual, quando o conhecimento abre espaço para novidades científicas e explosões, que são os momentos em que há um aumento significativo de publicação de trabalhos sobre o mesmo tema. O CiteSpace III é um programa exclusivamente para analisar as cocitações, adotando técnicas subjacentes as suas vezes de repetição, como um elemento para denominar as maiores incidências em citações (Pinto et al., 2006). As cocitações utilizadas pelo software relacionam autores, países, referencias, instituições, termo, palavra-chave, revistas e jornais.

O mecanismo da análise de citações funciona por meio de autores citam artigos que consideram importantes no desenvolvimento de suas pesquisas, dando a esses artigos em valor significativo. Akim e Mergulhão (2015) afirmam que quanto mais citado o trabalho for maior a sua influência sobre a área de estudo. Segundo Vanz e Caregnato (2003), essa análise pode ainda ser estendida à "contagem" das referências arroladas no final do texto, o que possibilita identificar e mapear características da comunicação científica, medir o impacto e a visibilidade de determinados autores dentro de uma comunidade científica e também verificar quais "escolas" do pensamento vigoram dentro das mesmas. É importante frisar, que a análise de citações não tem como princípio medir o número de vezes que um determinado autor está certo ou errado, mas, sim, medir o nível de contribuição de um pesquisador ou uma instituição à ciência (Garfield, 1979).

A análise de cocitação considera a relação e a frequência de documentos que são citados em outros documentos, essa relação é feita a cada dois documentos. A premissa fundamental dessa análise é que, quanto mais dois documentos são cocitados conjuntamente, maior é a probabilidade de que tenham conteúdos relacionados (Spinak, 1996). Assim, observa-se elementos gráficos nas representações das redes sociais, que mostram os conjuntos de autores e as ligações entre eles. Nas redes sociais também é possível analisar as citações e as cocitações. Para se obter uma boa análise das citações e cocitações dos artigos, as referências processadas no CiteSpace III exigem padronização para a eliminação de possíveis duplicidades que interferem no mapeamento da rede e no levantamento de indicadores como os de frequência, centralidade e densidade (Akim e Mergulhão, 2015). 
Os indicadores de frequência indicam quantas vezes um autor foi citado em um artigo. De acordo com Marteleto (2001), o indicador de centralidade é utilizado na análise de redes de cocitações e representa os atores que mais se relacionam com os demais; logo, um ator com grande centralidade está em contato direto e adjacente com muitos outros atores da rede, sendo reconhecido como um grande canal de informações, trazendo a ideia de poder. Hanneman e Riddle (2005) afirmam que o indicador de densidade exprime o grau de conectividade de uma rede de modo que, quanto mais densa ela for, maior é a possibilidade de fluxo de informações. Esse indicador é calculado tendo como numerador o número de relações existentes e denominador o número de relações possíveis.

Para a análise bibliométrica utilizando o software escolhido, foi necessário baixar novamente os artigos na base de dados da Web of Science (WoS) Institute for Scientific Information (ISI), agora no formato .txt, que é o formato lido pelo software. Essa base de dados conta com mais de 8400 periódicos de diferentes áreas, facilitando a realização da análise, pois fornece informações dos artigos citados pelos periódicos, o que propicia a criação de uma rede bibliográfica (Macedo, 2012). Dos 64 artigos que faziam parte do escopo do trabalho, apenas 60 foram encontrados na base de dados da WoS. Portanto, os artigos de Ansbro e Wang (2013), Jahre (1995), Fetter e Rakes (2012) e Beullens (2004) não foram incluídos ou analisados, apesar de terem sido identificados pelo escopo do trabalho.

Já a análise sistêmica consistiu de uma classificação dos artigos em relação ao assunto abordado por cada um deles, por meio do método de solução, variável tempo, abordagem, função objetivo, modelo e modelagem utilizada, definidos conforme revisão bibliográfica. Estes são baseados no contexto dos artigos revisados a fim de encontrar a categorização adequada para os mesmos. Cada classificação é subdividida em categorias especificas, essas foram escolhidas de acordo com a frequência com que o assunto foi abordado dentro da amostra.

Os resultados da análise bibliométrica, juntamente com o resultado da análise sistêmica comporão o estado da arte acerca do tema pesquisado.

\section{Resultados e Discussões}

\subsection{Análise Bibliométrica das Publicações}

A análise dos 60 artigos apontou que eles receberam um total 2765 citações até abril de 2015. A evolução das publicações da amostra é representada na Gráfico 1 e a evolução das citações é observada no Gráfico 2. A partir desses gráficos pode-se observar o aumento das publicações ao longo dos anos.

A partir de 2007 é observado um crescimento, chegando ao patamar de 9 publicações nos anos de 2011 e 2013, essa evolução parece colaborar para o aumento no número de citações de artigos sobre o assunto. Esse aumento nas publicações pode ter sido impulsionado pelo crescente interesse dos acadêmicos em entender os impactos que a logística reversa pode causar sobre a utilização dos modelos de localização. Impactos estes que podem ser sentidos no cenário econômico pelas empresas, governo e sociedade.

O aumento das publicações reflete na importância que a logística reversa possui cada vez mais na área global da indústria por causa dos fatores ambientais e empresariais. Planejamento e implementação de uma rede de logística reversa adequada poderia trazer mais lucro, satisfação do cliente, e um quadro social agradável para as empresas (Pishvaee et al., 2010).

Em relação às citações referentes aos 60 artigos analisados, verificou-se que em média cada artigo foi citado 47,63 vezes. O indicador $h$-index foi de 27 , o que significa que os 29 artigos mais citados tiveram pelo menos 27 citações cada um. 
Gráfico 1: Publicações entre 1995 e 2015.

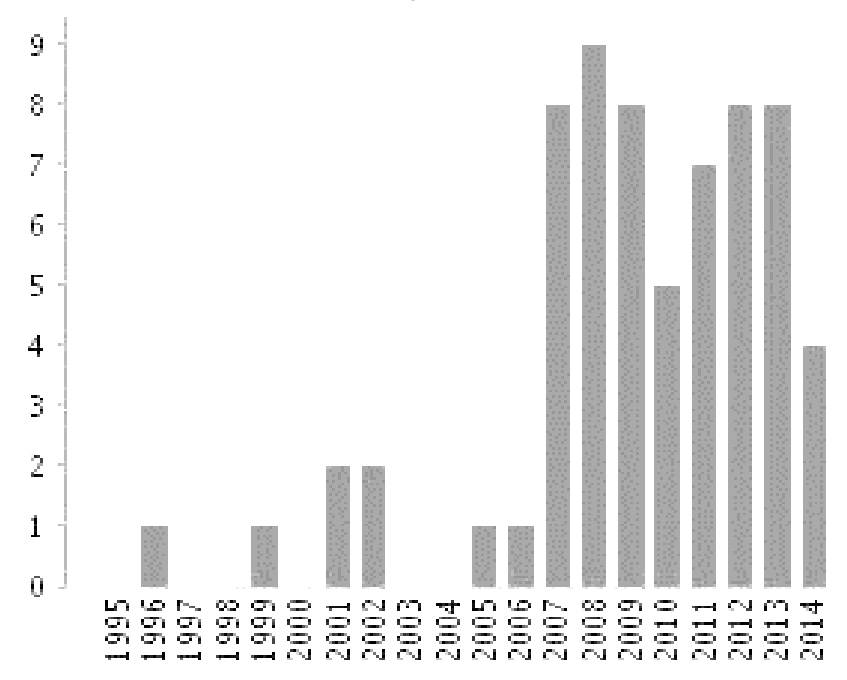

Fonte: Elaboração própria.

O Gráfico 2 mostra que a partir de 2000 começou um aumento no número de citações, evidenciando que o tema abordado passou a ter uma maior representação na academia. Somente no ano de 2011 houve uma interrupção neste aumento, que foi retomado no ano seguinte. Entre os anos de 2012 e 2014 houve um considerável aumento no número de citações nos periódicos publicados. No ano de 2015, verifica-se que o número de citações já passava de 500 somente até o mês de abril, o que permite supor que o aumento de citações deve ter continuado naquele ano.

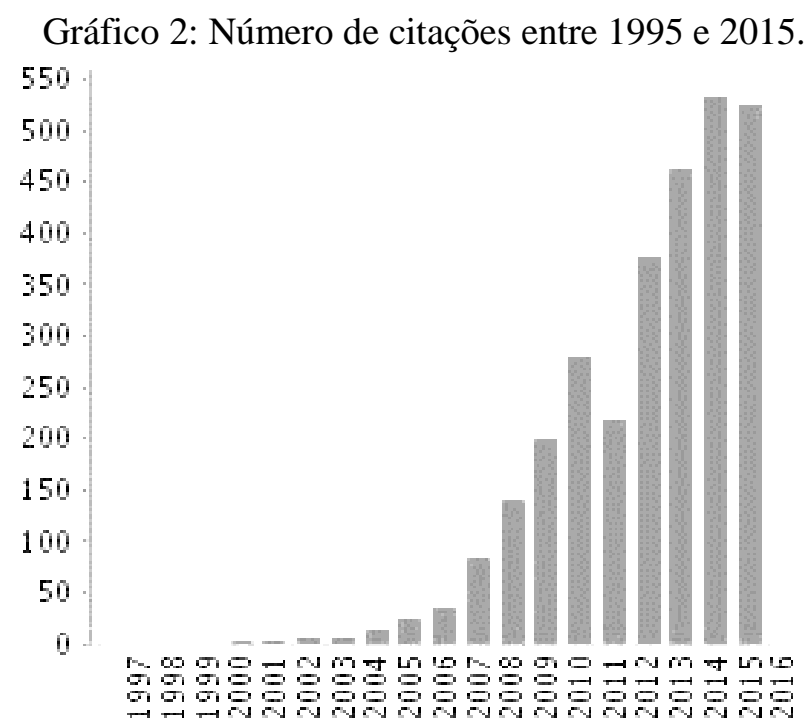

Fonte: Elaboração própria.

A Tabela 1 lista os 10 artigos mais citados dentre a amostra pesquisada. Os artigos abordam diferentes assuntos dentro do tema estudo. Dentre os artigos, alguns autores propõem estratégias para problemas de localização de instalação dentro de uma rede logística, outros dão ênfase a importância da remanufatura de produtos e a inclusão de produtos descartados no fluxo logístico.

A Tabela 2 mostra a média de citação por ano dos artigos com média acima de 10,00. Melo et al. (2009) possuem o artigo mais citado tanto em quantidade quanto em média, o que expressa maior credibilidade em seu trabalho. Porém, a ordem dos artigos mais citados não confere com a ordem dos artigos mais citados por ano. 
Tabela 1: Artigos mais citados no período de 1995 a 2015.

\begin{tabular}{|c|c|c|c|c|}
\hline Título & Ano & Autores & Periódico & $\begin{array}{l}\text { Cita } \\
\text { ções. }\end{array}$ \\
\hline $\begin{array}{l}\text { Facility location and supply chain } \\
\text { management - A review }\end{array}$ & 2009 & $\begin{array}{l}\text { Melo; } \\
\text { Nickel; } \\
\text { Saldanha- } \\
\text { da-Gama }\end{array}$ & $\begin{array}{l}\text { European Journal of } \\
\text { Operational Research }\end{array}$ & 305 \\
\hline $\begin{array}{l}\text { Facility location models for } \\
\text { distribution system design }\end{array}$ & 2005 & $\begin{array}{l}\text { Klose; } \\
\text { Drexl }\end{array}$ & $\begin{array}{l}\text { European Journal of } \\
\text { Operational Research }\end{array}$ & 197 \\
\hline $\begin{array}{l}\text { Reverse logistics system planning } \\
\text { for recycling electrical appliances } \\
\text { and computers in Taiwan }\end{array}$ & 2001 & Shih & $\begin{array}{l}\text { Resources } \\
\text { Conservation and } \\
\text { Recycling }\end{array}$ & 119 \\
\hline $\begin{array}{l}\text { A genetic algorithm-based heuristic } \\
\text { for the dynamic integrated } \\
\text { forward/reverse logistics network } \\
\text { for 3PLs }\end{array}$ & 2007 & Ko; Evans & $\begin{array}{c}\text { Computers \& } \\
\text { Operations Research }\end{array}$ & 116 \\
\hline $\begin{array}{l}\text { Network design for reverse } \\
\text { logistics }\end{array}$ & 2008 & Srivastava & $\begin{array}{l}\text { Omega-International } \\
\text { Journal of } \\
\text { Management Science }\end{array}$ & 114 \\
\hline $\begin{array}{l}\text { A genetic algorithm approach to } \\
\text { developing the multi-echelon } \\
\text { reverse logistics network for } \\
\text { product returns }\end{array}$ & 2006 & $\begin{array}{l}\text { Min; Ko; } \\
\quad \text { Ko }\end{array}$ & $\begin{array}{l}\text { Omega-International } \\
\text { Journal of } \\
\text { Management Science }\end{array}$ & 102 \\
\hline $\begin{array}{l}\text { A facility location model for } \\
\text { logistics systems including reverse } \\
\text { flows: The case of remanufacturing } \\
\text { activities }\end{array}$ & 2007 & Lu; Bostel & $\begin{array}{c}\text { Computers \& } \\
\text { Operations Research }\end{array}$ & 89 \\
\hline $\begin{array}{l}\text { A reverse logistics cost } \\
\text { minimization model for the } \\
\text { treatment of hazardous wastes }\end{array}$ & 2002 & $\begin{array}{l}\text { Hu; Sheu; } \\
\text { Huang }\end{array}$ & $\begin{array}{c}\text { Transportation } \\
\text { Research Part E- } \\
\text { Logistics and } \\
\text { Transportation Review }\end{array}$ & 80 \\
\hline $\begin{array}{l}\text { A facility location allocation model } \\
\text { for reusing carpet materials }\end{array}$ & 1999 & $\begin{array}{l}\text { Louwers; } \\
\text { Kip; Peters; } \\
\text { Souren; } \\
\text { Flapper }\end{array}$ & $\begin{array}{c}\text { Computers \& } \\
\text { Industrial Engineering }\end{array}$ & 72 \\
\hline $\begin{array}{l}\text { An integrated model of facility } \\
\text { location and transportation network } \\
\text { design }\end{array}$ & 2001 & $\begin{array}{l}\text { Melkote; } \\
\text { Daskin }\end{array}$ & $\begin{array}{c}\text { Transportation } \\
\text { Research Part A-Policy } \\
\text { and Practice }\end{array}$ & 67 \\
\hline
\end{tabular}

Fonte: Elaboração própria.

Os artigos coletados tiveram suas publicações apresentadas em 30 periódicos diferentes. A Tabela 3 apresenta os principais periódicos que mais publicaram no tema abordado. É possível verificar que a maior quantidade de publicações está nas revistas Resources, Conservation and Recycling e European Journal of Operational Research. A primeira revista aborda temas de aspectos tecnológicos, econômicos e políticas de gestão de recursos específicos como reciclagem, além de assuntos estratégicos como melhoria da produtividade da produção e seus recursos. A segunda revista é uma das revistas de referência mundial da área de Pesquisa Operacional e Logística. Dentre os periódicos listados, há o predomínio da literatura estrangeira. 
Tabela 2: Média das citações dos artigos por ano.

\begin{tabular}{|c|c|c|c|c|}
\hline Título & Ano & Autores & Periódico & Média \\
\hline $\begin{array}{l}\text { Facility location and supply chain } \\
\text { management - A review }\end{array}$ & 2009 & $\begin{array}{l}\text { Melo; } \\
\text { Nickel; } \\
\text { Saldanha- } \\
\text { da-Gama }\end{array}$ & $\begin{array}{l}\text { European Journal of } \\
\text { Operational Research }\end{array}$ & 43,86 \\
\hline $\begin{array}{l}\text { Facility location models for } \\
\text { distribution system design }\end{array}$ & 2005 & $\begin{array}{l}\text { Klose; } \\
\text { Drexl }\end{array}$ & $\begin{array}{l}\text { European Journal of } \\
\text { Operational Research }\end{array}$ & 18,00 \\
\hline $\begin{array}{l}\text { Network design for reverse } \\
\text { logistics }\end{array}$ & 2008 & Srivastava & $\begin{array}{l}\text { Omega-International } \\
\text { Journal of } \\
\text { Management Science }\end{array}$ & 14,38 \\
\hline $\begin{array}{l}\text { A genetic algorithm-based heuristic } \\
\text { for the dynamic integrated } \\
\text { forward/reverse logistics network } \\
\text { for 3PLs }\end{array}$ & 2007 & Ko; Evans & $\begin{array}{c}\text { Computers \& } \\
\text { Operations Research }\end{array}$ & 12,89 \\
\hline $\begin{array}{l}\text { A memetic algorithm for bi- } \\
\text { objective integrated } \\
\text { forward/reverse logistics network } \\
\text { design }\end{array}$ & 2010 & $\begin{array}{l}\text { Pishvaee; } \\
\text { Farahani; } \\
\text { Dullaert }\end{array}$ & $\begin{array}{c}\text { Computers \& } \\
\text { Operations Research }\end{array}$ & 11,00 \\
\hline $\begin{array}{l}\text { A genetic algorithm approach to } \\
\text { developing the multi-echelon } \\
\text { reverse logistics network for } \\
\text { product returns }\end{array}$ & 2006 & $\begin{array}{l}\text { Min; Ko; } \\
\text { Ko }\end{array}$ & $\begin{array}{l}\text { Omega-International } \\
\text { Journal of } \\
\text { Management Science }\end{array}$ & 10,20 \\
\hline
\end{tabular}

Fonte: Elaboração própria.

Tabela 3: Total de publicações nos periódicos mais publicados.

\begin{tabular}{|l|c|c|c|c|c|}
\hline \multicolumn{1}{|c|}{ Periódicos } & $\begin{array}{c}\mathbf{1 9 9 6} \\
\mathbf{a} \\
\mathbf{2 0 0 0}\end{array}$ & $\begin{array}{c}\mathbf{2 0 0 1} \mathbf{\mathbf { 2 }} \\
\mathbf{2 0 0 5}\end{array}$ & $\begin{array}{c}\mathbf{2 0 0 6} \\
\mathbf{2 0 1 0}\end{array}$ & $\begin{array}{c}\mathbf{2 0 1 1} \\
\mathbf{a} \\
\mathbf{2 0 1 5}\end{array}$ & $\begin{array}{c}\text { Total de } \\
\text { artigos } \\
\text { publicados }\end{array}$ \\
\hline Resources, Conservation and Recycling & 0 & 2 & 0 & 9 & 11 \\
\hline European Journal of Operational Research & 1 & 1 & 3 & 4 & 9 \\
\hline Computers \& Industrial Engineering & 1 & 0 & 3 & 3 & 7 \\
\hline Computers \& Operations Research & 0 & 0 & 7 & 0 & 7 \\
\hline $\begin{array}{l}\text { International Journal of Production } \\
\text { Economics }\end{array}$ & 0 & 1 & 5 & 0 & 6 \\
\hline $\begin{array}{l}\text { Omega-International Journal of Management } \\
\text { Science }\end{array}$ & 0 & 0 & 2 & 2 & 4 \\
\hline $\begin{array}{l}\text { International Journal of Advanced } \\
\text { Manufacturing Technology }\end{array}$ & 0 & 0 & 3 & 0 & 3 \\
\hline Outros & 1 & 3 & 9 & 15 & 28 \\
\hline
\end{tabular}

Fonte: Elaboração própria.

O período de 2011 a 2015 é o responsável pelo maior número de publicações nos periódicos, 45,5\% da amostra, seguido do período de 2006 a 2010 com 42,4\% dos artigos da amostra. Essas informações confirmam os dados do Gráfico 1 que mostra a ascendência do tema a partir do ano de 2007.

Outra analise refere-se a rede de colaboração entre os coautores dos artigos da amostra. A Figura 2 mostra uma rede com 195 nós, ou seja, autores, e possui densidade de 0,0112, indicando que apenas $1,12 \%$ de todas as conexões possíveis foram estabelecidas, o que mostra pouca conexão entre os autores. A rede apresenta 66 clusters, o que indica que existem 66 agrupamentos de nós. Quanto mais clusters existem menos conectados estão os autores, ou seja, 
existem muitos grupos de autores que se relacionam entre si, mas com pouca interação entre eles.

Figura 2: Rede completa de autores cocitados.

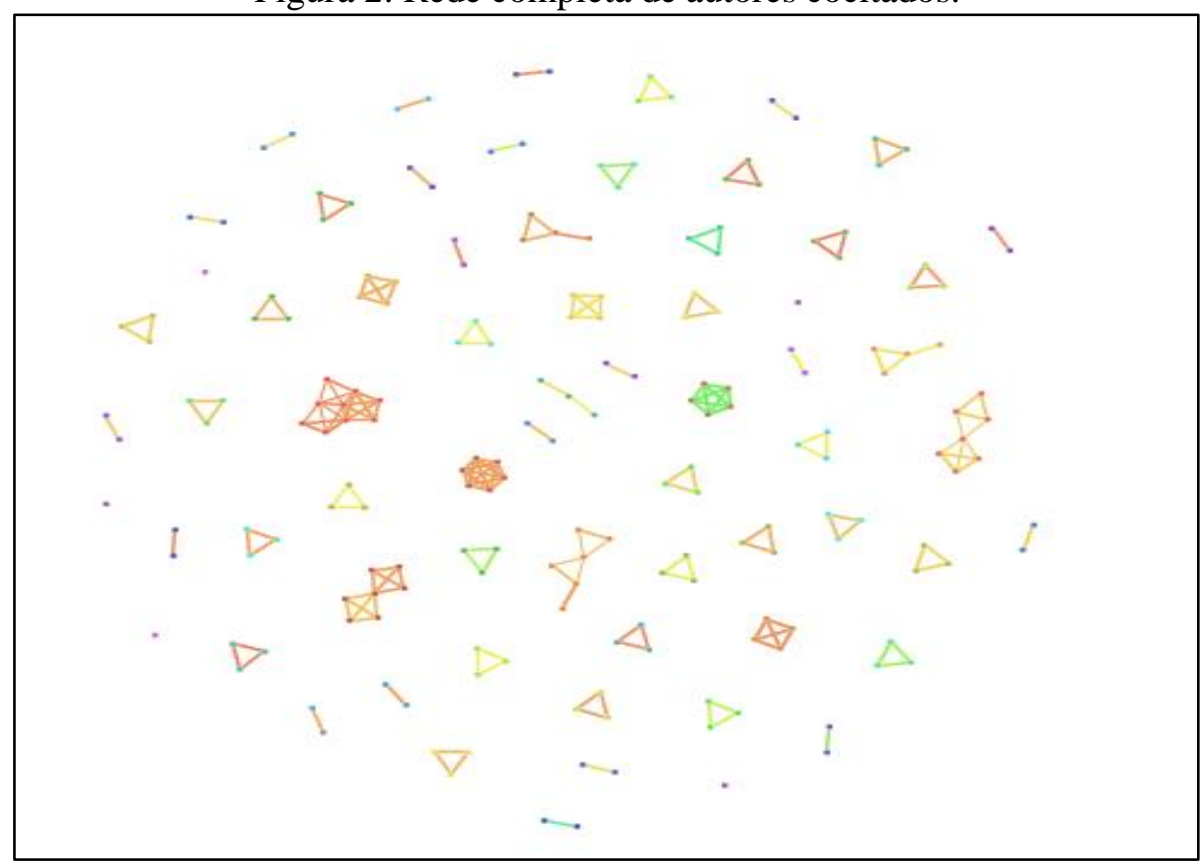

Fonte: Elaboração própria a partir do CiteSpace II.

Nota-se um aglomerado mais evidente, o que significa uma concentração maior de autores cocitados, conforme Figura 3. Pode-se identificar uma centralidade na representação dos autores Diabat, Kannan e Svetinovic. Cada nó possui um tamanho diferente, o que indica o número de publicações.

Em relação à frequência, que indica quantas vezes um autor foi citado em um artigo, mais uma vez são destacados os mesmos autores, porque no período estudado estes autores foram os mais importantes, sendo identificados também outros autores importantes para os estudos de logística reversa e modelos de localização, como Govindan et al. (2015). Há autores como Abdallah que não tem uma grande representação, mas se converte em um ponto de ligação entre estudiosos.

Figura 3: Analise da principal cluster.

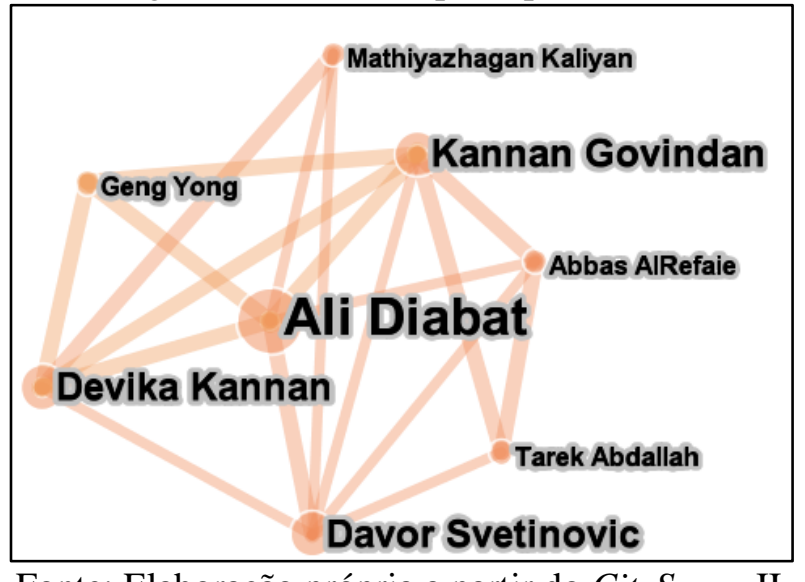

Fonte: Elaboração própria a partir do CiteSpace II. 
Na Tabela 4, observa-se que os Estados Unidos é o país que possui maior frequência, o que significa que há muitas publicações de autores de instituições norte americanas, e o Canadá o país de maior centralidade, relatando que ele possui mais autores ou instituições que publicam mais com outros países.

Por intermédio da Figura 4, observa-se que o Canadá é o país que mais possui conexões com outros países (quatro), o que indica que possui maior densidade sendo que sua ligação mais forte é com a Turquia, podendo haver parcerias entre esses países por meio de instituições ou autores referentes a abordagem sobre modelos de localização e logística reversa. Países como EUA, Inglaterra, Espanha e Emirados Árabes possuem importante representatividade no assunto abordado com algumas interações.

Tabela 4: Classificação dos 10 países de maior frequência.

\begin{tabular}{|c|c|c|}
\hline País & Frequência & Centralidade \\
\hline EUA & 16 & 0.41 \\
\hline Canadá & 8 & 0.34 \\
\hline Turquia & 6 & 0.09 \\
\hline Taiwan & 5 & 0.00 \\
\hline China & 5 & 0.09 \\
\hline Portugal & 5 & 0.09 \\
\hline Iran & 5 & 0.00 \\
\hline Coreia do Sul & 4 & 0.09 \\
\hline Espanha & 4 & 0.23 \\
\hline Holanda & 4 & 0.00 \\
\hline
\end{tabular}

Fonte: Elaboração própria a partir do CiteSpace II.

Cada anel representa um segmento de tempo a partir da primeira publicação. As cores representam o ano de publicação do artigo, seguindo uma escala de cor: as cores mais frias, que estão mais ao centro dos nós, expõem as publicações mais antigas e as cores mais quentes que são mais externas representam as mais recentes. Essa dinâmica é observada na Figura 4. Os anéis rosa na parte externa diferenciam os países com maior centralidade, logo podemos observar-se que o Canadá e os EUA possuem os anéis rosa mais destacados, seguido da Espanha e da Inglaterra.

Nesta rede, percebe-se algumas parcerias de destaque entre países, como uma forte ligação entre os USA e a Coreia do Sul, notada pela espessura da ligação. A cor esverdeada indica que essa parceria ocorreu entre 2005 e 2009. Foi neste intervalo de tempo que o assunto abordado neste trabalhou começou sua ascensão, como mostrado no Gráfico 2. Os EUA também possuem uma relação forte e mais recente com a Inglaterra, meados do ano de 2013.

Uma relação entre países mais recente é vista entre o Canadá e a Turquia, que pela escala de cor representa os artigos publicados do ano de 2013 até hoje. Outra relação recente, por volta do ano de 2013, é evidenciada entre os países Emirados Árabes e Índia.

Grécia, Egito, Polônia, Suíça, Eslovênia, França, Holanda, Austrália e Servia são os países que possuem nenhuma ligação com nenhum outro país, ou seja, eles não possuem densidade. 
Figura 4: Mapa de relacionamento entre os países.

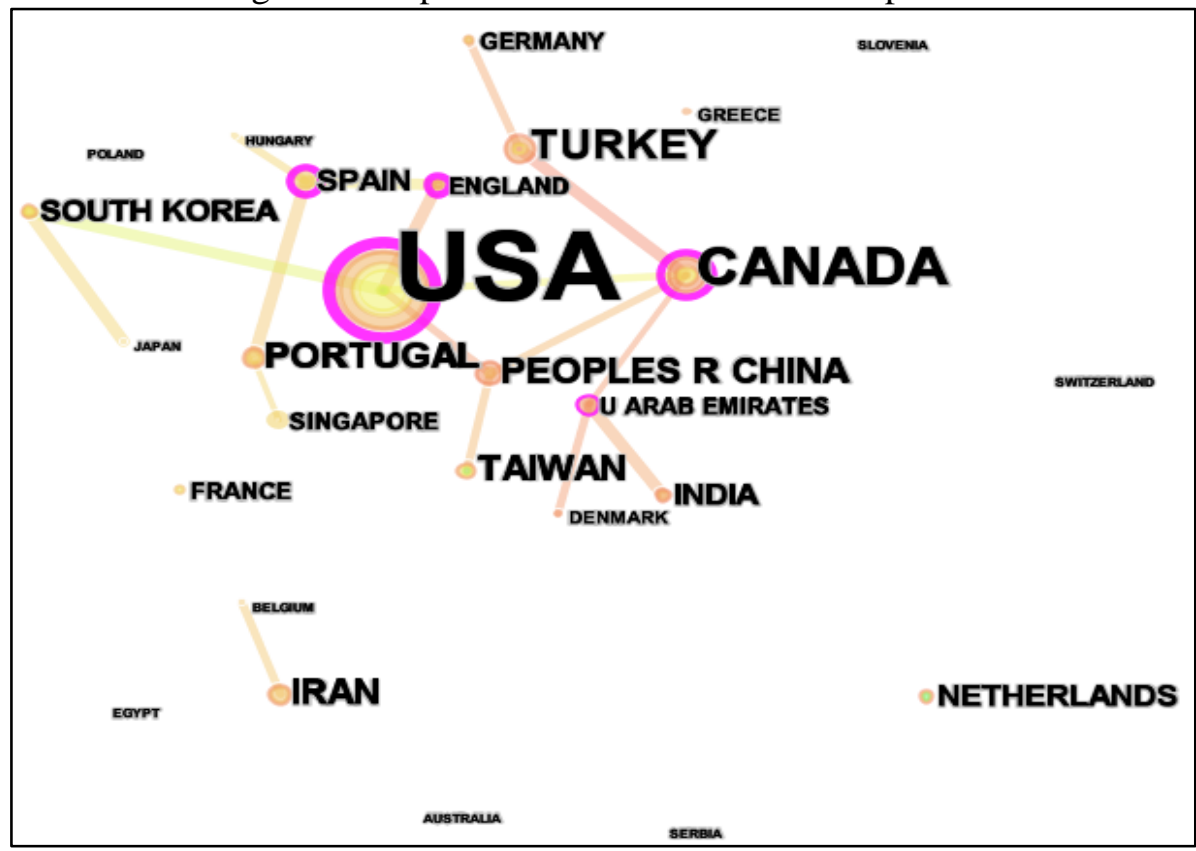

Fonte: Elaboração própria a partir do CiteSpace II.

\subsection{Análise Sistêmica das Publicações}

Esta análise se inicia com uma leitura aprofundada das publicações que compõem a amostra. A próxima etapa envolve a realização de um diagnóstico detalhado da utilização dos modelos de localização pelos 60 artigos analisados nesse trabalho, por diferentes óticas. Um motivo impulsionador do uso de um modelo de localização é escolher o porquê da utilização do mesmo e a sua meta.

As categorias escolhidas pelas quais os artigos foram classificados encontram-se na Tabela 5. Estes são baseados em diferentes aspectos dos artigos revisados, na tentativa de encontrar categorização adequada para todos os documentos. Quanto às subdivisões das categorias, elas foram escolhidas de acordo com a frequência com que o assunto foi abordado dentro da amostra, cada subdivisão deve conter uma quantidade suficiente de artigos analisados.

Foram consideradas as principais dimensões dentro de cada categoria. Na metodologia de resolução, encontram-se solucionadores exatos, metodologia heurística (por aproximação) ou trabalhos que utilizaram os dois métodos. A variável tempo pode variar entre um modelo estático, ou seja, não dependente da variável tempo; ou um modelo é dinâmico, para os dados que variam com o tempo.

A abordagem dos estudos pode ser real ou estimada, ou seja, o primeiro caso aborda os trabalhos que tiveram aplicação prática em casos reais, e o segundo caso refere-se a trabalhos que se restringem apenas a estudos sem aplicação. A função objetivo procura a solução final de uma pesquisa. $\mathrm{O}$ trabalho que almeja uma solução ótima e única, busca um único objetivo, podendo ser esse de maximização ou de minimização, já os trabalhos que oferecem mais de uma solução, possuem uma função multiobjetivo.

Para auxiliar na decisão da localização de facilidades e análise de demanda, existem diversos modelos com estas funções, sendo os mais abordados na amostra estudada os modelos de cobertura, p-centro, p-mediana e dispersão. Para este trabalho, as ferramentas ou modelagens de significativa importância são: Sistemas de Informação Geográfica (SIG), Programação Inteira (PI), Programação Inteira Binária (PIB), Programação Linear Inteira Mista (PLIM), Programação Não-Linear Inteira Mista (PNLIM) e Algoritmo Fuzzy. 
Tabela 5: Classificação dos artigos analisados por categorias.

\begin{tabular}{|l|l|l|l|l|l|}
\hline \multicolumn{1}{|c|}{$\begin{array}{c}\text { Método de } \\
\text { solução }\end{array}$} & $\begin{array}{c}\text { Variável } \\
\text { tempo }\end{array}$ & Abordagem & $\begin{array}{c}\text { Função } \\
\text { objetivo }\end{array}$ & Modelo & Modelagem \\
\hline Exata & Dinâmico & Real & Único objetivo & Cobertura & SIG \\
\hline Heurística & Estático & Estimada & Multiobjectivo & p-Centro & PLI \\
\hline $\begin{array}{l}\text { Exata e } \\
\text { Heurística }\end{array}$ & & & & p-Mediana & PIB \\
\hline & & & & Não se aplica & PLIM \\
\hline & & & & & PNLIM \\
\hline & & & & & Fuzzy \\
\hline & & & & & Não se aplica \\
\hline
\end{tabular}

Fonte: Elaboração própria.

Deve ser salientado que estas classificações são as principais categorias, escolhidas por suas maiores frequências na amostra. Porém, em cada categoria foram observadas outras dimensões que poderiam ser abordadas, mas não estavam presentes em muitos artigos. A classificação das categorias de 57 dos 60 artigos da amostra estudada encontra-se no Apêndice A.

No decorrer da análise sistêmica da amostra dos artigos, foram descobertos dois artigos que não utilizaram nenhum dos modelos abordadas neste trabalho. A primeira refere-se ao modelo Data Envelopment Analysis (DEA), cuja abordagem foi desenvolvida por Charnes et al. (1978) para determinar a eficiência de unidades produtivas, onde não seja relevante ou não se deseja considerar somente o aspecto financeiro. O DEA permite avaliar a eficiência relativa considerando-se os recursos inputs e os outputs alcançados. O segundo é o general algebraic modeling system (GAMS) que constitui uma ferramenta com capacidade para a determinação de soluções ótimas, tanto em problemas lineares como nos casos de não lineares, permitindo, inclusive, trabalhar-se com grandes números de variáveis, constantes e restrições (Da Paz e De Silans, 2002).

Três artigos não foram incorporados nesta classificação, pois são artigos de revisão bibliográfica acerca do tema. Melo et al. (2009) propõem uma revisão bibliográfica sobre localização de facilidades e gestão da cadeia de suprimentos com fluxos reversos e diretos. Arabani e Farahani (2012), realizaram uma revisão sobre os conceitos da dinâmica de localização de facilidades, classificando e avaliando diversos artigos em categorias determinadas. Klose e Drexl (2005) revisaram algumas contribuições sobre o design do sistema de distribuição estratégica para as empresas, que possuem como problema a localização de instalações e alocação de clientes.

A análise dos Gráficos 3 e 4 permitiu observar que a modelagem mais utilizada pelos artigos da amostra foi a PLIM, com 44,9\% dos artigos. Dentre os modelos mais utilizados, a pmediana se destacou com $48,3 \%$ dos artigos. A relação entre a modelagem PLIM e o modelo de p-mediana merece destaque, pois mais da metade dos problemas que envolviam p-mediana foram solucionadas por meio da PLIM. Essa relação já era esperada, já que segundo Longley e Batty (1996) e Vallim Filho (2004), a PLIM é o método matemático mais empregado para resolução de problemas de localização formulados segundo o modelo das medianas, conduzindo para a solução matemática ótima do problema.

O Gráfico 3 ilustra a quantidade de artigos por modelagem utilizada. Apesar da modelagem SIG ter grande importância para a análise e apresentação de dados e auxilio para localização de facilidades (Neto e Da Silva Lima, 2006), foi encontrado apenas um artigo que utilizou essa ferramenta para resolução do modelo matemático. Os artigos que não foram possíveis identificar qual modelagem foi utilizada contabilizam 10,3\% dos artigos da amostra. 
Gráfico 3: Quantidade de artigos por modelagem.

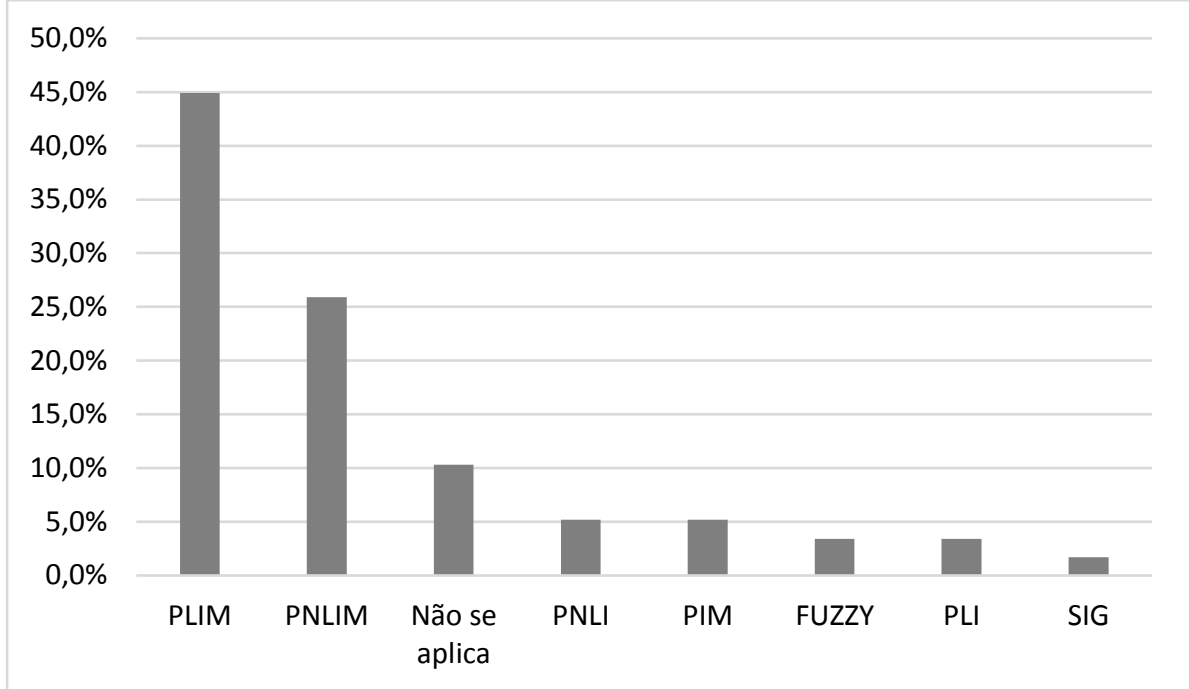

Fonte: Elaboração própria.

O Gráfico 4 mostra a distribuição dos artigos pelos principais modelos matemáticos abordados neste trabalho. Vale ressaltar que a classificação dos artigos pelos modelos é difícil pois, na prática, para solução dos problemas, os modelos são alterados e acabam sendo derivações dos modelos classificados. Foram encontrados em 7 artigos da amostra com a formulação de algoritmos genéticos. Segundo Linden (2008), os algoritmos genéticos são uma técnica de busca extremamente eficiente no seu objetivo de varrer o espaço de soluções e encontrar soluções próximas da solução ótima, quase sem necessitar da interferência humana, sendo uma técnica de inteligência computacional. São mais adequados para problemas especialmente difíceis. Os modelos DEA e GAMS só foram utilizados uma vez cada um.

Mesmo com tantos modelos existentes na literatura, alguns artigos não possuíram nenhuma classificação quanto aos mesmos. Alguns artigos não foram explícitos quanto a utilização do método, impossibilitando a compreensão do mesmo. Outro motivo que também pode ter interferido nessa classificação da amostra, foi a falta de conhecimento de outras abordagens mais especificas que foram utilizadas por alguns autores.

Gráfico 4: Quantidade de artigos por modelo matemático.

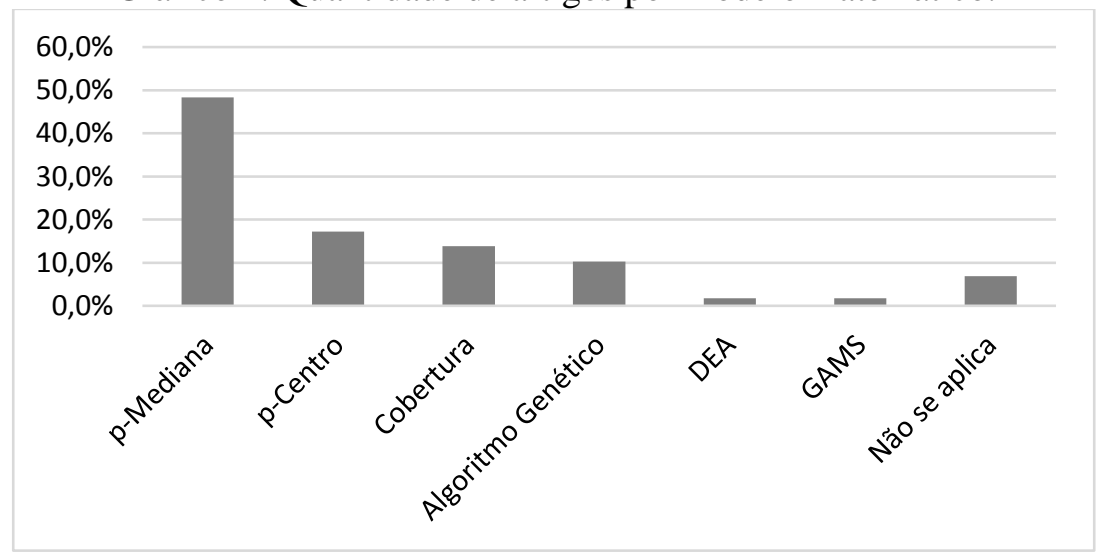

Fonte: Elaboração própria.

Do total de artigos analisados, $56,1 \%$ dos artigos apresentavam funções multiobjectivo. Desses, $64,5 \%$ dos artigos utilizaram o modelo p-mediana para encontrar suas soluções. Costa (2014) destaca que um dos principais motivos que impulsionam a análise de redes é a determinação de localização de facilidades que reduza custos por meio dos modelos de mediana. 
Em toda amostra, apenas quatro artigos utilizaram os métodos heurísticos e exatos simultaneamente para resolver suas pesquisas. Os artigos que utilizaram heurísticas como método de solução de seus trabalhos equivalem a $71,4 \%$, sendo que a maioria destes trabalhos envolveram instâncias estimadas. Apenas $38,1 \%$ dos artigos aplicaram seus resultados em casos reais.

Os artigos que levaram em consideração a variável "tempo computacional" correspondem a $65,1 \%$ da amostra. A complexidade da modelagem matemática aumenta consideravelmente quando esta variável é levada em consideração em uma pesquisa. Apenas 31,8\% dos artigos da amostra aplicaram em casos reais os modelos matemáticos que avaliaram o tempo como restrição.

\subsection{Análise das Pesquisas Futuras das Publicações}

Esta seção apresenta uma síntese das pesquisas futuras apontadas pelos autores que propuseram sugestões em seus trabalhos. Apenas os artigos a partir do ano de 2007 foram verificados, pois foi após esse ano que as publicações começaram a ter um aumento significativo. Dentre os 57 artigos examinados na análise sistêmica, apenas 23 autores fizeram algum tipo de sugestão futura. Dentre as principais recomendações feitas pelos autores, a sugestão mais ressaltada refere-se à aplicação dos modelos matemáticos propostos em casos reais, ou seja, referem-se a artigos que testaram seus modelos com instâncias aleatórias.

A utilização da variável "tempo computacional" também foi apontada como sugestão, principalmente pela sua relação com os casos reais. Sahyouni et al. (2007), Amin e Zhang (2013), Wang et al. (2007), Diabat et al. (2013), Min et al. (2006), Pishvaee et al. (2010), CruzRivera e Ertel (2009), Hu e Sheu (2013) frisaram que para conquistar um modelo matemático adequado aplicado em cenários reais, é necessário considerar a variável tempo, para que o modelo seja aplicado de maneira a incorporar alterações ao longo do tempo, conforme ocorre na realidade. Os autores Coutinho-Rodrigues et al. (2012), Kannan et al. (2012), Diabat et al. (2013), Pishvaee et al. (2010), Cardoso et al. (2013), Suyabatmaz et al. (2014) sugerem outra direção para pesquisas futuras, que incluem a formulação de modelos multiobjetivos, ou seja, que incorpore outra abordagem ao problema. Na prática, é muito difícil que um problema real tenha um único objetivo, mas para facilitar ou possibilitar a análise, trabalha-se com um único objetivo. A utilização de métodos heurísticos também foi sugerida, devido à complexidade de alguns algoritmos.

A maioria dos autores citados destacam a importância da extensão de suas pesquisas por outras, que entre alguns motivos como falta de informação e de recursos não puderam realizar completamente as propostas em seus trabalhos.

Pode-se concluir que os autores que não destacaram nenhuma sugestão futura, alcançaram seus resultados e objetivos propostos ou pretendem, eles mesmos, realizarem o aprimoramento de seus trabalhos futuramente.

\section{Considerações Finais}

A presente pesquisa apresentou os artigos que dão ênfase ao estudo dos modelos de localização de facilidade com aplicação na logística reversa encontradas no Portal Capes. Foram analisados 60 artigos, sendo o primeiro publicado em 1994. Porém, o tema teve um aumento expressivo a partir de 2007, chegando a 9 publicações nos anos de 2011 e 2013.

Em relação as citações, a partir de 2000 começou um aumento de seus números, evidenciando que o tema abordado passou a ter uma maior representação na academia. Sabendo que a pesquisa foi realizada em abril de 2015 , verifica-se que o número de citações já passa de 500 , o que nos faz supor que o aumento de citações se continuará neste ano, o que demostra que o campo de pesquisa é recente e emergente.

Os autores Melo et al. (2009) possuem o artigo mais citado tanto em quantidade quanto em média, o que expressa maior credibilidade em seu trabalho. Dentre os 30 periódicos envolvidos nesta pesquisa, a revista de maior destaque em relação à temática é a Resources, 
Conservation and Recycling. As parcerias de pesquisa que estão concentradas com maior destaque nos EUA e no Canadá, que são também os países que mais publicam sobre o tema.

Pode-se concluir a literatura sobre modelos de localização de facilidade com aplicação na logística reversa é progressiva no meio acadêmico mundial. Percebe-se que esse aumento vem a partir dos estudos que envolvem a concepção da rede de logística reversa, sendo que a principal decisão envolvida se refere à localização de facilidades locais. Entretanto, esta pesquisa se baseou na base de dados Portal da Capes. Sugere-se que outras pesquisas de comparação com estudos bibliométricos de outras bases de dados podem ser apropriados para uma avaliação mais completa.

Essa pesquisa possibilita compreender a importância do tema abordado em diferentes situações, seja ela em industrias com preocupações ambientais ou com anseio de lucros, bem como a melhor escolha de instalações e sua capacidade. Os resultados contribuem para ressaltar a importância das parcerias de pesquisa entre países como forma de enriquecer as discussões e fomentar publicações de qualidade como aquelas da base Web of Science.

Agradecimentos. Os autores agradecem o apoio fornecido pelo Conselho Nacional de Desenvolvimento Científico e Tecnológico - CNPq (Processo no 311664 / 2015-6). Os autores agradecem aos revisores anônimos por seus valiosos comentários.

\section{Referências}

Aboolian, R., Berman, O. e Krass, D. Competitive facility location and design problem. European Journal of Operational Research, v. 182, n. 1, p. 40-62, 2007.

Ahluwalia, P. K. e Nema, A. K. Capacity planning for electronic waste management facilities under uncertainty: multi-objective multi-time-step model development. Waste Management \& Research, v. 29, n. 7, p. 694-709, 2011.

Akim, E. K. e Mergulhão, R. C. Panorama da produção intelectual sobre a medição de desempenho na gestão pública. Revista de Administração Pública, v. 49, n. 2, p. 337-366, 2015.

Alumur, S. A., Nickel, S., Saldanha-da-Gama, F. e Verter, V. Multi-period reverse logistics network design. European Journal of Operational Research, v. 220, n. 1, p. 67-78, 2012.

Amin, S. H. e Zhang, G. A multi-objective facility location model for closed-loop supply chain network under uncertain demand and return. Applied Mathematical Modelling, v. 37, n. 6, p. 4165-4176, 2013.

Ansbro, D. e Wang, Q. A facility location model for socio-environmentally responsible decision-making. Journal of Remanufacturing, v. 3, n. 1, p. 5, 2013.

Arabani, A. B. e Farahani, R. Z. Facility location dynamics: An overview of classifications and applications. Computers \& Industrial Engineering, v. 62, n. 1, p. 408-420, 2012.

Barker, T. J. e Zabinsky, Z. B. A multicriteria decision making model for reverse logistics using analytical hierarchy process. Omega, v. 39, n. 5, p. 558-573, 2011.

Beullens, P. Reverse logistics in effective recovery of products from waste materials. Reviews in Environmental Science and Biotechnology, v. 3, n. 4, p. 283-306, 2004.

Biehl, M., Prater, E. e Realff, M. J. Assessing performance and uncertainty in developing carpet reverse logistics systems. Computers \& Operations Research, v. 34, n. 2, p. 443-463, 2007. 
Bing, X., Bloemhof-Ruwaard, J. M. e Van der Vorst, J. G. A. J. Sustainable reverse logistics network design for household plastic waste. Flexible Services and Manufacturing Journal, v. 26, n. 1-2, p. 119-142, 2014.

Bloemhof-Ruwaard, J. M., Salomon, M. e Van Wassenhove, L. N. The capacitated distribution and waste disposal problem. European Journal of Operational Research, v. 88, n. 3, p. 490-503, 1996.

Boffey, T. B., Mesa, J. A., Ortega, F. A. e Rodrigues, J. I. Locating a low-level waste disposal site. Computers \& Operations Research, v. 35, n. 3, p. 701-716, 2008.

Brimberg, J., Juel, H. e Schöbel, A. Linear facility location in three dimensions-models and solution methods. Operations Research, v. 50, n. 6, p. 1050-1057, 2002.

Cardoso, S. R., Barbosa-Póvoa, A. P. F. D. e Relvas, S. Design and planning of supply chains with integration of reverse logistics activities under demand uncertainty. European Journal of Operational Research, v. 226, n. 3, p. 436-451, 2013.

Cardoso, R. C. V., Souza, E. V. A. e Santos, P. Q. Unidades de alimentação e nutrição nos campi da Universidade Federal da Bahia: um estudo sob a perspectiva do alimento seguro. Revista de Nutrição, v. 18, n. 5, p. 669-680, 2005.

Charnes, A., Cooper, W. W. e Rhodes, E. Measuring the efficiency of decision making units. European Journal of Operational Research, v. 2, n. 6, p. 429-444, 1978.

Chen, C. CiteSpace II: Detecting and visualizing emerging trends and transient patterns in scientific literature. Journal of the American Society for Information Science and Technology, v. 57, n. 3, p. 359-377, 2006.

Chen, H.-K., Chou, H.-W. e Chiu, Y-C. On the modeling and solution algorithm for the reverse logistics recycling flow equilibrium problem. Transportation Research Part C: Emerging Technologies, v. 15, n. 4, p. 218-234, 2007.

Costa, M. B. B. Utilização de modelo de localização-alocação para identificação de zoneamento logístico integrado ao planejamento estratégico de transportes. $224 \mathrm{f}$. Tese (Doutorado em Engenharia de Transportes) - COPPE, Universidade Federal do Rio de Janeiro, Rio de Janeiro-RJ, 2014.

Coutinho-Rodrigues, J., Tralhão, L. e Alçada-Almeida, L. A bi-objective modeling approach applied to an urban semi-desirable facility location problem. European Journal of Operational Research, v. 223, n. 1, p. 203-213, 2012.

Cruz-Rivera, R. e Ertel, J. Reverse logistics network design for the collection of end-of-life vehicles in Mexico. European Journal of Operational Research, v. 196, n. 3, p. 930-939, 2009.

Da Paz, A. R. e De Silans, A. M. B. P. Aplicação do GAMS (General Algebraic Modeling System) a um sistema de reservatórios e captação na bacia hidrográfica do Rio Gramame. João Pessoa-PB: Editora Universitária UFPB, p. 189-196, 2002.

De Brito, M. P. Managing reverse logistics or reversing logistics management? Erasmus Research Institute of Management (ERIM). 2004. Disponível em: <http://hdl.handle.net/1765/1132>. Acesso em: 28/05/2015. 
Demirel, N. Ö. e Gökçen, H. A mixed integer programming model for remanufacturing in reverse logistics environment. The International Journal of Advanced Manufacturing Technology, v. 39, n. 11-12, p. 1197-1206, 2008.

Diabat, A., Abdallah, T., Al-Refaie, A., Svetinovic, D. e Govindan, K. Strategic closed-loop facility location problem with carbon market trading. IEEE Transactions on Engineering Management, v. 60, n. 2, p. 398-408, 2013.

Drezner, Z. e Hamacher, H. W. (Eds.). Facility Location: Applications and Theory. Springer Science \& Business Media, 2002.

Du, F. e Evans, G. W. A bi-objective reverse logistics network analysis for post-sale service. Computers \& Operations Research, v. 35, n. 8, p. 2617-2634, 2008.

El Korchi, A. e Millet, D. Designing a sustainable reverse logistics channel: the 18 generic structures framework. Journal of Cleaner Production, v. 19, n. 6-7, p. 588-597, 2011.

El-Sayed, M., Afia, N. e El-Kharbotly, A. A stochastic model for forward-reverse logistics network design under risk. Computers \& Industrial Engineering, v. 58, n. 3, p. 423-431, 2010.

Ferri, G. L., Chaves, G. L. D. e Ribeiro, G. M. Reverse logistics network for municipal solid waste management: The inclusion of waste pickers as a Brazilian legal requirement. Waste Management, v. 40, p. 173-191, 2015.

Fetter, G. e Rakes, T. Incorporating recycling into post-disaster debris disposal. Socio-Economic Planning Sciences, v. 46, n. 1, p. 14-22, 2012.

Fleischmann, M., Bloemhof-Ruwaard, J. M., Dekker, R., Van der Laan, E., Van Nunen, J. A. E. E. e Van Wassenhove, L. N. Quantitative models for reverse logistics: A review. European Journal of Operational Research, v. 103, n. 1, p. 1-17, 1997.

Fonseca, M. C., García-Sánchez, A., Ortega-Mier, M. e Saldanha-da-Gama, F. A stochastic biobjective location model for strategic reverse logistics. Top, v. 18, n. 1, p. 158-184, 2010.

Fuller, D. A. e Allen, J. A typology of reverse channel systems for post-consumer recyclables. In: Polonsky, M. J. e Mintu-Wimsatt, A. T. (Eds.), Environmental Marketing: Strategies, Practice, Theory, and Research, cap. 12, p. 241-266. Haworth Press, 1995.

Garfield, E. Is citation analysis a legitimate evaluation tool? Scientometrics, v. 1, n. 4, p. 359$375,1979$.

Gen, M., Cheng, R. e Lin, L. Network models and optimization: Multiobjective genetic algorithm approach. London: Springer Science \& Business Media, 2008.

Ghiani, G., Laganà, D., Manni, E., Musmanno, R. e Vigo, D. Operations research in solid waste management: a survey of strategic and tactical issues. Computers \& Operations Research, v. 44, p. 22-32, 2014.

Gołębiewski, B., Trajer, J., Jaros, M. e Winiczenko, R. Modelling of the location of vehicle recycling facilities: A case study in Poland. Resources, Conservation and Recycling, v. 80, p. 10-20, 2013. 
Govindan, K., Diabat, A. e Popiuc, M. N. Contract analysis: A performance measures and profit evaluation within two-echelon supply chains. Computers \& Industrial Engineering, v. 63, n. 1, p. 58-74, 2012.

Govindan, K., Soleimani, H. e Kannan, D. Reverse logistics and closed-loop supply chain: A comprehensive review to explore the future. European Journal of Operational Research, v. 240, n. 3, p. 603-626, 2015.

Hanneman, R. A. e Riddle, M. Introduction to Social Network Methods. Riverside, CA: University of California, 2005.

Hu, Z.-H. e Sheu, J.-B. Post-disaster debris reverse logistics management under psychological cost minimization. Transportation Research Part B: Methodological, v. 55, p. 118-141, 2013.

Hu, T.-L., Sheu, J.-B. e Huang, K.-H. A reverse logistics cost minimization model for the treatment of hazardous wastes. Transportation Research Part E: Logistics and Transportation Review, v. 38, n. 6, p. 457-473, 2002.

Jahre, M. Household waste collection as a reverse channel: A theoretical perspective. International Journal of Physical Distribution \& Logistics Management, v. 25, n. 2, p. 39-55, 1995.

Kannan, D., Diabat, A., Alrefaei, M., Govindan, K. e Yong, G. A carbon footprint based reverse logistics network design model. Resources, Conservation and Recycling, v. 67, p. 75-79, 2012.

Kara, S., Rugrungruang, F. e Kaebernick, H. Simulation modelling of reverse logistics networks. International Journal of Production Economics, v. 106, n. 1, p. 61-69, 2007.

Kaya, I. e Cinar, D. Facility location selection using a fuzzy outranking method. In: Proceedings of the $7^{\text {th }}$ International FLINS Conference, Genova, Italy. World Scientific, 2006. p. 359-366.

Khadivi, M. R. e Ghomi, S. M. T. F. Solid waste facilities location using of analytical network process and data envelopment analysis approaches. Waste Management, v. 32, n. 6, p. 1258$1265,2012$.

Kim, J.-S., e Lee, D.-H. A restricted dynamic model for refuse collection network design in reverse logistics. Computers \& Industrial Engineering, v. 66, n. 4, p. 1131-1137, 2013.

Klose, A. e Drexl, A. Facility location models for distribution system design. European Journal of Operational Research, v. 162, n. 1, p. 4-29, 2005.

Ko, H. J. e Evans, G. W. A genetic algorithm-based heuristic for the dynamic integrated forward/reverse logistics network for 3PLs. Computers \& Operations Research, v. 34, n. 2, p. 346-366, 2007.

Kobashi, N. Y. e Santos, R. N. M. Arqueologia do trabalho imaterial: uma aplicação bibliométrica à análise de dissertações e teses. Encontros Bibli: Revista Eletrônica de Biblioteconomia e Ciência da Informação, v. 13, n. 1, p. 106-115, 2008.

Lacerda, L. Logística Reversa: Uma visão sobre os conceitos e as práticas operacionais. In: Figueiredo, K. F., Fleury, P. F. e Wanke, P. (Orgs.) Logística e Gerenciamento da Cadeia de Suprimentos: planejamento do fluxo de produtos e dos recursos. Centro de Estudos em Logística. COPPEAD, UFRJ. São Paulo: Atlas, 2003. 
Lee, D.-H. e Dong, M. Dynamic network design for reverse logistics operations under uncertainty. Transportation Research Part E: Logistics and Transportation Review, v. 45, n. 1, p. 61-71, 2009.

Lee, J.-E., Gen, M. e Rhee, K.-G. Network model and optimization of reverse logistics by hybrid genetic algorithm. Computers \& Industrial Engineering, v. 56, n. 3, p. 951-964, 2009.

Leeds, S. Data mining: Beware of the shaft. Direct Marketing, v. 62, n. 9, p. 38-42, 2000.

Leite, P. R. Logística Reversa: Meio Ambiente e Competitividade. $2^{\mathrm{a}}$ ed. Pearson Prentice Hall, 2009.

Leite Filho, G. A. e Siqueira, R. L. Revista contabilidade \& finanças USP: Uma análise bibliométrica de 1999 a 2006. Revista de Informação Contábil, v. 1, n. 2, p. 102-119, 2007.

Linden, R. Algoritmos Genéticos: Uma importante ferramenta da Inteligência Computacional. $2^{\mathrm{a}}$ ed. Rio de Janeiro: Brasport, 2008.

Liu, D. Network site optimization of reverse logistics for E-commerce based on genetic algorithm. Neural Computing and Applications, v. 25, n. 1, p. 67-71, 2014.

Longley, P. A. e Batty, M. (Eds.) Spatial Analysis: Modelling in a GIS Environment. John Wiley \& Sons, 1996.

Louwers, D., Kip, B. J., Peters, E., Souren, F. e Flapper, S. D. P. A facility location allocation model for reusing carpet materials. Computers \& Industrial Engineering, v. 36, n. 4, p. 855-869, 1999.

Lu, Z. e Bostel, N. A facility location model for logistics systems including reverse flows: The case of remanufacturing activities. Computers \& Operations Research, v. 34, n. 2, p. 299-323, 2007.

Macedo, N. L. F. Fatores que afetam o uso da medição de desempenho em indústrias de laticínios: um estudo multicascos. 117 f. Dissertação (Mestrado em Engenharia de Produção) Universidade Federal de São Carlos - UFSCAR, Sorocaba-SP, 2012.

Macias-Chapula, C. A. O papel da informetria e da cienciometria e sua perspectiva nacional e internacional. Ciência da Informação, v. 27, n. 2, p. 134-140, 1998.

Mahmoudzadeh, M., Mansour, S. e Karimi, B. To develop a third-party reverse logistics network for end-of-life vehicles in Iran. Resources, Conservation and Recycling, v. 78, p. 1-14, 2013.

Mansour, S. e Zarei, M. A multi-period reverse logistics optimisation model for end-of-life vehicles recovery based on EU Directive. International Journal of Computer Integrated Manufacturing, v. 21, n. 7, p. 764-777, 2008.

Marteleto, R. M. Análise de redes sociais - aplicação nos estudos de transferência da informação. Ciência da Informação, v. 30, n. 1, p. 71-81, 2001.

Melkote, S. e Daskin, M. S. An integrated model of facility location and transportation network design. Transportation Research Part A: Policy and Practice, v. 35, n. 6, p. 515-538, 2001. 
Melo, M. T., Nickel, S. e Saldanha-da-Gama, F. Facility location and supply chain management - A review. European Journal of Operational Research, v. 196, n. 2, p. 401-412. 2009.

Min, H., Ko, H. J. e Ko, C. S. A genetic algorithm approach to developing the multi-echelon reverse logistics network for product returns. Omega, v. 34, n. 1, p. 56-69, 2006.

Min, H. e Ko, H. The dynamic design of a reverse logistics network from the perspective of third-party logistics service providers. International Journal of Production Economics, v. 113, n. 1, p. 176-192, 2008.

Mutha, A. e Pokharel, S. Strategic network design for reverse logistics and remanufacturing using new and old product modules. Computers \& Industrial Engineering, v. 56, n. 1, p. 334346, 2009.

Neto, A. F. e Da Silva Lima, R. Roteirização de veículos de uma rede atacadista com o auxílio de Sistemas de Informações Geográficas (SIG). Revista Pesquisa e Desenvolvimento Engenharia de Produção, n. 5, p. 18-39, 2006.

Or, I. e Akgül, M. An optimization approach for locating a hazardous waste disposal facility in Istanbul Province. Waste Management \& Research, v. 12, n. 6, p. 495-506, 1994.

Penteado Filho, R. C., Faria, L. I. L., Vieira, J. L. G., Kurihara, M. H., Avila, A. F. D. e Quoniam, L. Aplicação da bibliometria na construção de indicadores sobre a produção científica da Embrapa. In: Anais do $3^{\circ}$ Workshop Brasileiro de Inteligência Competitiva e Gestão do Conhecimento, São Paulo, 2002.

Pinto, A. L., Rodríguez Barquín, B.-A. e Moreiro González, J. A. Análisis de citación de la revista Ciência da Informação del Ibict. Ciência da Informação, v. 35, n. 3, p. 153-165, 2006.

Pishvaee, M. S., Farahani, R. Z. e Dullaert, W. A memetic algorithm for bi-objective integrated forward/reverse logistics network design. Computers \& Operations Research, v. 37, n. 6, p. $1100-1112,2010$.

Pishvaee, M. S.; Kianfar, K. e Karimi, B. Reverse logistics network design using simulated annealing. The International Journal of Advanced Manufacturing Technology, v. 47, n. 1-4, p. 269-281, 2010.

Portal de Periódicos. CAPES/MEC. Disponível em: <http://www.periodicos.capes.gov.br/>. Acesso em: 09/05/2015.

Ramezani, M., Bashiri, M. e Tavakkoli-Moghaddam, R. A new multi-objective stochastic model for a forward/reverse logistic network design with responsiveness and quality level. Applied Mathematical Modelling, v. 37, n. 1-2, p. 328-344, 2013.

Rogers, D. S. e Tibben-Lembke, R. S. Going Backwards: Reverse Logistics Trends and Practices. Pittsburgh, PA: Reverse Logistics Executive Council, 1999.

Sahyouni, K., Savaskan, R. C. e Daskin, M. S. A facility location model for bidirectional flows. Transportation Science, v. 41, n. 4, p. 484-499, 2007.

Senthil, S., Srirangacharyulu, B. e Ramesh, A. A robust hybrid multi-criteria decision making methodology for contractor evaluation and selection in third-party reverse logistics. Expert Systems with Applications, v. 41, n. 1, p. 50-58, 2014. 
Shi, J., Zhang, G. e Sha, J. Optimal production and pricing policy for a closed loop system. Resources, Conservation and Recycling, v. 55, n. 6, p. 639-647, 2011.

Shih, L.-H. Reverse logistics system planning for recycling electrical appliances and computers in Taiwan. Resources, Conservation and Recycling, v. 32, n. 1, p. 55-72, 2001.

Spinak, E. Diccionario Enciclopédico de Bibliometría, Cienciometría e Informetría. Montevidéu: Unesco, 1996.

Srivastava, S. K. Network design for reverse logistics. Omega, v. 36, n. 4, p. 535-548, 2008.

Stadtler, H. Supply chain management and advanced planning-basics, overview and challenges. European Journal of Operational Research, v. 163, n. 3, p. 575-588, 2005.

Suyabatmaz, A. Ç., Altekin, F. T. e Şahin, G. Hybrid simulation-analytical modeling approaches for the reverse logistics network design of a third-party logistics provider. Computers \& Industrial Engineering, v. 70, p. 74-89, 2014.

Thanh, P. N., Bostel, N. e Péton, O. A dynamic model for facility location in the design of complex supply chains. International Journal of Production Economics, v. 113, n. 2, p. 678693, 2008.

Vallim Filho, A. R. A. Localização de centros de distribuição de carga: Contribuições à modelagem matemática. Tese (Doutorado em Engenharia de Transportes) - Escola Politécnica da Universidade de São Paulo, São Paulo-SP, 2004.

Vanz, S. A. S. e Caregnato, S. E. Estudos de citação: uma ferramenta para entender a comunicação científica. Em Questão, v. 9, n. 2, p. 295-307, 2003.

Vidovic, M., Dimitrijevic, B., Ratkovic, B. e Simic, V. A novel covering approach to positioning ELV collection points. Resources, Conservation and Recycling, v. 57, p. 1-9, 2011.

Wang, Z., Yao, D.-Q. e Huang, P. A new location-inventory policy with reverse logistics applied to B2C e-markets of China. International Journal of Production Economics, v. 107, n. 2, p. 350-363, 2007.

Wollenweber, J. A multi-stage facility location problem with staircase costs and splitting of commodities: model, heuristic approach and application. OR Spectrum, v. 30, n. 4, p. 655-673, 2008 .

Ye, L., Ye, C. e Chuang, Y.-F. Location set covering for waste resource recycling centers in Taiwan. Resources, Conservation and Recycling, v. 55, n. 11, p. 979-985, 2011.

Zhang, F., Johnson, D. M. e Sutherland, J. W. A GIS-based method for identifying the optimal location for a facility to convert forest biomass to biofuel. Biomass and Bioenergy, v. 35, n. 9, p. 3951-3961, 2011. 


\section{Apêndice A}

Tabela 6: Resumo das classificações de cada artigo por categoria

\begin{tabular}{|c|c|c|c|c|c|c|}
\hline Autores & $\begin{array}{l}\text { Método } \\
\text { de } \\
\text { solução }\end{array}$ & $\begin{array}{c}\text { Variável } \\
\text { tempo }\end{array}$ & Abordagem & $\begin{array}{l}\text { Função } \\
\text { objetivo }\end{array}$ & Modelo & Modelagem \\
\hline $\begin{array}{c}\text { Aboolian et al. } \\
(2007)\end{array}$ & $\mathrm{HEU}$ & ESTA & ESTI & MULTI & P-CENT & PLIM \\
\hline $\begin{array}{c}\text { Ahluwalia e } \\
\text { Nema (2011) }\end{array}$ & $\mathrm{HEU}$ & DIN & ESTI & MULTI & $\mathrm{AG}$ & PLIM \\
\hline $\begin{array}{l}\text { Alumur et al. } \\
(2012)\end{array}$ & $\mathrm{HEU}$ & DIN & REAL & $\mathrm{BI}$ & P-MED & PLIM \\
\hline $\begin{array}{l}\text { Amin e Zhang } \\
(2013)\end{array}$ & $\mathrm{HEU}$ & ESTA & ESTI & MULTI & P-MED & PLIM \\
\hline $\begin{array}{c}\text { Barker e } \\
\text { Zabinsky } \\
(2011)\end{array}$ & $\mathrm{HEU}$ & ESTA & REAL & MULTI & NA & NA \\
\hline $\begin{array}{l}\text { Biehl et al. } \\
(2007)\end{array}$ & $\mathrm{HEU}$ & DIN & REAL & MULTI & P-CENT & PLIM \\
\hline $\begin{array}{l}\text { Bing et al. } \\
\text { (2014) }\end{array}$ & HEU & ESTA & ESTI & MULTI & P-MED & PLIM \\
\hline $\begin{array}{c}\text { Bloemhof- } \\
\text { Ruwaard et al. } \\
(1996)\end{array}$ & EXA/HEU & ESTA & ESTI & UNI & P-MED & PLIM \\
\hline $\begin{array}{c}\text { Boffey et al. } \\
(2008)\end{array}$ & $\mathrm{HEU}$ & ESTA & REAL & MULTI & P-MED & NA \\
\hline $\begin{array}{c}\text { Brimberg et al. } \\
(2002)\end{array}$ & $\mathrm{HEU}$ & ESTA & DIN & MULTI & P-CENT & PNLI \\
\hline $\begin{array}{c}\text { Cardoso et al. } \\
(2013)\end{array}$ & $\mathrm{HEU}$ & ESTA & REAL & MULTI & P-MED & PLIM \\
\hline $\begin{array}{c}\text { Chen et al. } \\
(2007)\end{array}$ & $\mathrm{HEU}$ & ESTA & ESTI & MULTI & P-CEN & PNLIM \\
\hline $\begin{array}{c}\text { Coutinho- } \\
\text { Rodrigues et al. } \\
(2012)\end{array}$ & HEU & DIN & REAL & MULTI & P-MED & PLIM \\
\hline $\begin{array}{c}\text { Cruz-Rivera e } \\
\text { Ertel (2009) }\end{array}$ & HEU & DIN & REAL & UNI & P-MED & NA \\
\hline $\begin{array}{c}\text { Demirel e } \\
\text { Gökçen (2008) }\end{array}$ & HEU & ESTA & ESTI & UNI & $\mathrm{COB}$ & PLIM \\
\hline $\begin{array}{c}\text { Diabat et al. } \\
(2013)\end{array}$ & HEU & ESTA & REAL & MULTI & $\mathrm{AG}$ & PNLIM \\
\hline $\begin{array}{l}\text { Govindan et al. } \\
\text { (2012) }\end{array}$ & $\mathrm{HEU}$ & ESTA & ESTI & MULTI & P-MED & PNLIM \\
\hline
\end{tabular}

Fonte: Própria

Abreviaturas: EXA.: Exata, HEU.: Heurística, DIN.: Dinâmico, ESTA.: Estático, ESTI.: 1:1048576 UNI.: Único, COB.: Cobertura, CENT.: Centro, MED.: Mediana, NA: Não se aplica 
Tabela 7: Resumo das classificações de cada artigo por categoria (continuação)

\begin{tabular}{|c|c|c|c|c|c|c|}
\hline Autores & $\begin{array}{c}\text { Método } \\
\text { de } \\
\text { solução }\end{array}$ & $\begin{array}{c}\text { Variável } \\
\text { tempo }\end{array}$ & Abordagem & $\begin{array}{c}\text { Função } \\
\text { objetivo }\end{array}$ & Modelo & Modelagem \\
\hline $\begin{array}{c}\text { Du e Evans } \\
\text { (2008) }\end{array}$ & HEU & DIN & EST & MULTI & P-MED & PLIM \\
\hline $\begin{array}{l}\text { El Korchi e } \\
\text { Millet (2011) }\end{array}$ & HEU & ESTA & REAL & UNI & NA & NA \\
\hline $\begin{array}{c}\text { El-Sayed et al. } \\
(2010)\end{array}$ & EXA & DIN & ESTI & UNI & P-MED & PLIM \\
\hline $\begin{array}{c}\text { Fonseca et al. } \\
(2010)\end{array}$ & EXA & ESTA & ESTI & MULTI & P-MED & PLIM \\
\hline $\begin{array}{c}\text { Gen et al. } \\
\text { (2008) }\end{array}$ & HEU & ESTA & ESTI & UNI & $\mathrm{GA}$ & NA \\
\hline $\begin{array}{l}\text { Gołebiewsky } \\
\text { et al. (2013) }\end{array}$ & HEU & ESTA & REAL & MULTI & GA & PLIM \\
\hline $\begin{array}{l}\text { Hu et al. } \\
\text { (2002) }\end{array}$ & EXA & DIN & ESTI & UNI & P-MED & PLI \\
\hline $\begin{array}{l}\text { Hu e Sheu } \\
\text { (2013) }\end{array}$ & HEU & DIN & ESTI & MULTI & P-MED & PLIM \\
\hline $\begin{array}{c}\text { Kannan et al. } \\
(2012)\end{array}$ & HEU & DIN & REAL & UNI & P-MED & PLIM \\
\hline $\begin{array}{c}\text { Kara et al. } \\
(2007)\end{array}$ & EXA & ESTA & REAL & UNI & $\mathrm{COB}$ & PIM \\
\hline $\begin{array}{l}\text { Kaya e Cinar } \\
\text { (2006) }\end{array}$ & EXA & ESTA & REAL & UNI & $\mathrm{COB}$ & FUZZY \\
\hline $\begin{array}{c}\text { Khadivi e } \\
\text { Ghomi (2012) }\end{array}$ & HEU & ESTA & ESTI & UNI & DEA & PLI \\
\hline $\begin{array}{l}\text { Kim e Lee } \\
\text { (2013) }\end{array}$ & HEU & DIN & ESTI & MULTI & P-MED & PNLIM \\
\hline $\begin{array}{l}\text { Ko e Evans } \\
\text { (2007) }\end{array}$ & $\begin{array}{c}\text { EXA/HE } \\
\mathrm{U}\end{array}$ & DIN & ESTI & MULTI & P-MED & PNLIM \\
\hline $\begin{array}{l}\text { Lee et al. } \\
\text { (2009) }\end{array}$ & HEU & ESTA & ESTI & MULTI & $\mathrm{COB}$ & PNLIM \\
\hline $\begin{array}{l}\text { Lee e Dong } \\
\text { (2008) }\end{array}$ & HEU & DIN & ESTI & MULTI & $\mathrm{COB}$ & PNLIM \\
\hline Liu (2014) & HEU & ESTA & REAL & UNI & GA & PLIM \\
\hline $\begin{array}{c}\text { Louwers et al. } \\
\text { (1999) }\end{array}$ & EXA & DIN & REAL & UNI & P-MED & PNLIM \\
\hline $\begin{array}{l}\text { Lu e Bostel } \\
\text { (2007) }\end{array}$ & HEU & DIN & ESTI & UNI & P-CENT & PNLIM \\
\hline $\begin{array}{l}\text { Mahmoudzade } \\
\text { h et al. (2013) }\end{array}$ & HEU & ESTA & ESTI & UNI & P-CENT & PLIM \\
\hline $\begin{array}{c}\text { Mansour e } \\
\text { Zarei (2008) }\end{array}$ & HEU & DIN & ESTI & MULTI & P-CENT & PLIM \\
\hline $\begin{array}{c}\text { Melkote e } \\
\text { Daskin (2001) }\end{array}$ & HEU & DIN & ESTI & MULTI & P-MED & PIM \\
\hline
\end{tabular}

Fonte: Própria

Abreviaturas: EXA.: Exata, HEU.: Heurística, DIN.: Dinâmico, ESTA.: Estático, ESTI.: 1:1048576 UNI.: Único, COB.: Cobertura, CENT.: Centro, MED.: Mediana, NA: Não se aplica 
Tabela 8: Resumo das classificações de cada artigo por categoria (conclusão)

\begin{tabular}{|c|c|c|c|c|c|c|}
\hline Autores & $\begin{array}{c}\text { Método } \\
\text { de } \\
\text { solução }\end{array}$ & $\begin{array}{c}\text { Variável } \\
\text { tempo }\end{array}$ & Abordagem & $\begin{array}{c}\text { Função } \\
\text { objetivo }\end{array}$ & Modelo & Modelagem \\
\hline $\begin{array}{c}\text { Min et al. } \\
(2006)\end{array}$ & HEU & DIN & ESTI & MULTI & P-MED & PNLIM \\
\hline $\begin{array}{c}\text { Min e Ko. } \\
(2008)\end{array}$ & HEU & ESTA & ESTI & UNI & P-CENT & PLIM \\
\hline $\begin{array}{c}\text { Mutha e } \\
\text { Pokharel } \\
(2009)\end{array}$ & HEU & DIN & ESTI & UNI & P-MED & PNLIM \\
\hline $\begin{array}{c}\text { Or e Akgul } \\
(1994)\end{array}$ & HEU & ESTA & REAL & UNI & P-CENT & PNLIM \\
\hline $\begin{array}{c}\text { Pishvaee; } \\
\text { Farahani et al. } \\
(2010)\end{array}$ & EXA & ESTA & ESTI & MULTI & P-MED & PLIM \\
\hline $\begin{array}{c}\text { Pishvaee; } \\
\text { Kianfar et al. } \\
(2010)\end{array}$ & EXA & DIN & ESTI & MULTI & P-MED & PLIM \\
\hline $\begin{array}{c}\text { Sahyouni et al. } \\
(2007)\end{array}$ & HEU & ESTA & ESTI & MULTI & P-CENT & PNLIM \\
\hline $\begin{array}{c}\text { Senthil et al. } \\
(2014)\end{array}$ & EXA/HE & ESTA & ESTI & UNI & NA & FUZZY \\
\hline $\begin{array}{c}\text { Shi et al. } \\
(2011)\end{array}$ & HEU & ESTA & ESTI & UNI & P-MED & PLIM \\
\hline $\begin{array}{c}\text { Shih (2001) } \\
\text { Srivastava } \\
(2008)\end{array}$ & HEU & ESTA & REAL & UNI & COB & PIM \\
\hline $\begin{array}{c}\text { Suyabatmaz } \\
\text { et al. (2014) }\end{array}$ & HEU & ESTA & ESTI & MULTI & AG & PLIM \\
\hline $\begin{array}{c}\text { Thanh et al. } \\
(2008)\end{array}$ & HEU & DIN & REAL & MULTI & P-MED & PLIM \\
\hline $\begin{array}{c}\text { Vidovic et al. } \\
(2011)\end{array}$ & HEU & ESTA & REAL & UNI & COB & PLIM \\
\hline $\begin{array}{c}\text { Wang et al. } \\
(2007)\end{array}$ & HEU & ESTA & ESTI & UNI & COB & PNLI \\
\hline $\begin{array}{c}\text { Wollenweber } \\
(2008)\end{array}$ & HEU & ESTA & ESTI & UNI & P-MED & PNLIM \\
\hline $\begin{array}{c}\text { Ye et al. } \\
(2011)\end{array}$ & HEU & ESTA & REAL & MULTI & P-MED & NA \\
\hline
\end{tabular}

Fonte: Própria

Abreviaturas: EXA.: Exata, HEU.: Heurística, DIN.: Dinâmico, ESTA.: Estático, ESTI.: 1:1048576 UNI.: Único, COB.: Cobertura, CENT.: Centro, MED.: Mediana, NA: Não se aplica 